\title{
Measuring Heavy Metals Content in Wastewater using Laser Induced Breakdown Spectroscopy
}

\author{
S. Ali ${ }^{1, \star}, \mathbf{H}$. Jamil ${ }^{2}$, M. Imran \\ ${ }^{1}$ Department of Physics, University of Gujrat Hafiz Hayat Campus, 50700 Gujrat, Pakistan \\ ${ }^{2}$ Department of Physics, University of Engineering and Technology, 54890 Lahore, Pakistan \\ *E-mail address: safdar.ali@uog.edu.pk
}

\begin{abstract}
In the present study, laser-induced breakdown spectroscopy (LIBS) has been applied for the determination of chromium and cadmium contents in wastewater of different canneries surrounding by leather factories. The plasma was generated with a Q-switched Nd:YAG laser having a fundamental wavelength of $1064 \mathrm{~nm}$ and delivers maximum pulse energy of $180 \mathrm{~mJ}$. Experimental conditions were optimized to improve the sensitivity and to get a reasonable signal from the generated plasma. The results show that the concentration of chromium and cadmium are varying in different samples depending on the incoming flow of the wastewater from factories. The results from LIBS and standard analytical technique called flame atomic absorption spectroscopy (FAAS) were compared and found in good agreement within the experimental uncertainties.
\end{abstract}

Keywords: heavy metals; leather factories; wastewater; spectroscopy

\section{INTRODUCTION}

There has been an increasing interest on environmental problems from last few decades. Many efforts are in progress towards the development of analytical techniques suitable for monitoring the concentration of pollutants and health hazards material in many kinds of environmental media, including soil, minerals, water and air [1]. Among the potential wastewater pollutants, heavy metals such as cadmium and chromium are important and are present in the waste of industry.

These elements cause serious health effects to tannery workers and the local communities as wastes goes into tanneries without proper treatment and tanneries water are then used in the cultivation of crops and vegetables. This work is a small effort to determine the amount of cadmium and chromium in the wastewater running in the canneries of Narowal, Sialkot and Gujrat cities of Pakistan.

Laser-induced breakdown spectroscopy (LIBS) is a type of simple atomic emission spectroscopy technique useful for the analysis of various samples of environmental and geological interest [2]. Due to the formation of laser plasma in aerosols, slurries, solids, gases, liquids LIBS can be applied to determine the elemental compositions of a wide range of materials in the solid, liquid or gaseous phase.

In this technique, a high power laser pulse is used to create plasma on the sample surface, resulting vaporization and atomization of a small amount of target material to form fast growing plasma cloud. 
The spectroscopic emission from the plasma allows for the identification of elements within the sample [3]. LIBS has rapidly become an important analytical technique to provide information about different atoms present in the sample.

In general atomic absorption spectroscopy (AAS) and UV-Visible spectrophotometry is used to study heavy metals in the industrial wastage [4].

The drawback of these congenital analytical techniques is the sample preparation, which consumes lot of time. Both these analytical techniques require time-consuming sample preparation protocols $[5,6]$.

LIBS on the other hand have various advantages over more conventional methods of atomic emission spectroscopy. LIBS, for example provide a direct analysis of solid samples and does not required any sample preparation [7].

Moreover, for real-time, in-situ site characterization on can apply LIBS technique. These advantages make the LIBS technique particularly interesting to determine the pollutants contents in wastewater, soil and environmental analysis.

In this paper we report about the chromium and cadmium contents found in the cannery wastewater of Gujrat, Sialkot and Narowal cities of Pakistan. In addition to the LIBS analysis of the metal contents of the selected samples, flame atomic absorption spectrometry (FAAS) were also used and the results were compared.

\section{EXPERIMENTAL SET UP}

The schematic diagram of the LIBS system used in the present study is shown in figure 1. A Q-switched Nd:YAG laser was used for the excitation with a fundamental wavelength of $1064 \mathrm{~nm}$ and delivers maximum pulse energy of $180 \mathrm{~mJ}$ with pulse duration of $10 \mathrm{~ns}$ and runs at a $10 \mathrm{~Hz}$ repetition rate.

The LIBS2500plus spectrometer [8] is used which allow to study the spectra in the range of 200-980 $\mathrm{nm}$. A convex lens of $100 \mathrm{~mm}$ focal length is used for focusing of the laser beam on the delay time between the laser and spectrometer we used delay gate 535 (DG 535).

The spectrometer generates a pulse to excite the laser which then triggers DG 535. After a delay time the spectrometer is triggered by the DG 535 and start collecting data. The plasma is generated at the repatriation rate of the laser pulses, i.e. at 10 times per second.

The x-rays emitted from the sample surface are collected by using a 2-m long multimode fibre optics. The transmitted light through the fibre is then reached at the other end of the fibre where multichannel spectrometer is connected.

The software called OOILIBS [8] is used to display and identify the emission spectra. The data pulses from the spectrometer are then stored in the computer.

The LIBS parameters were optimized to get high emission and collection rate. We optimize the following four parameters in the present study; laser pulse energy, delay time lens to sample distance, fibre optics (light collection system) distance from the sample. The optimization was carried out with the standards samples of chromium and cadmium. 


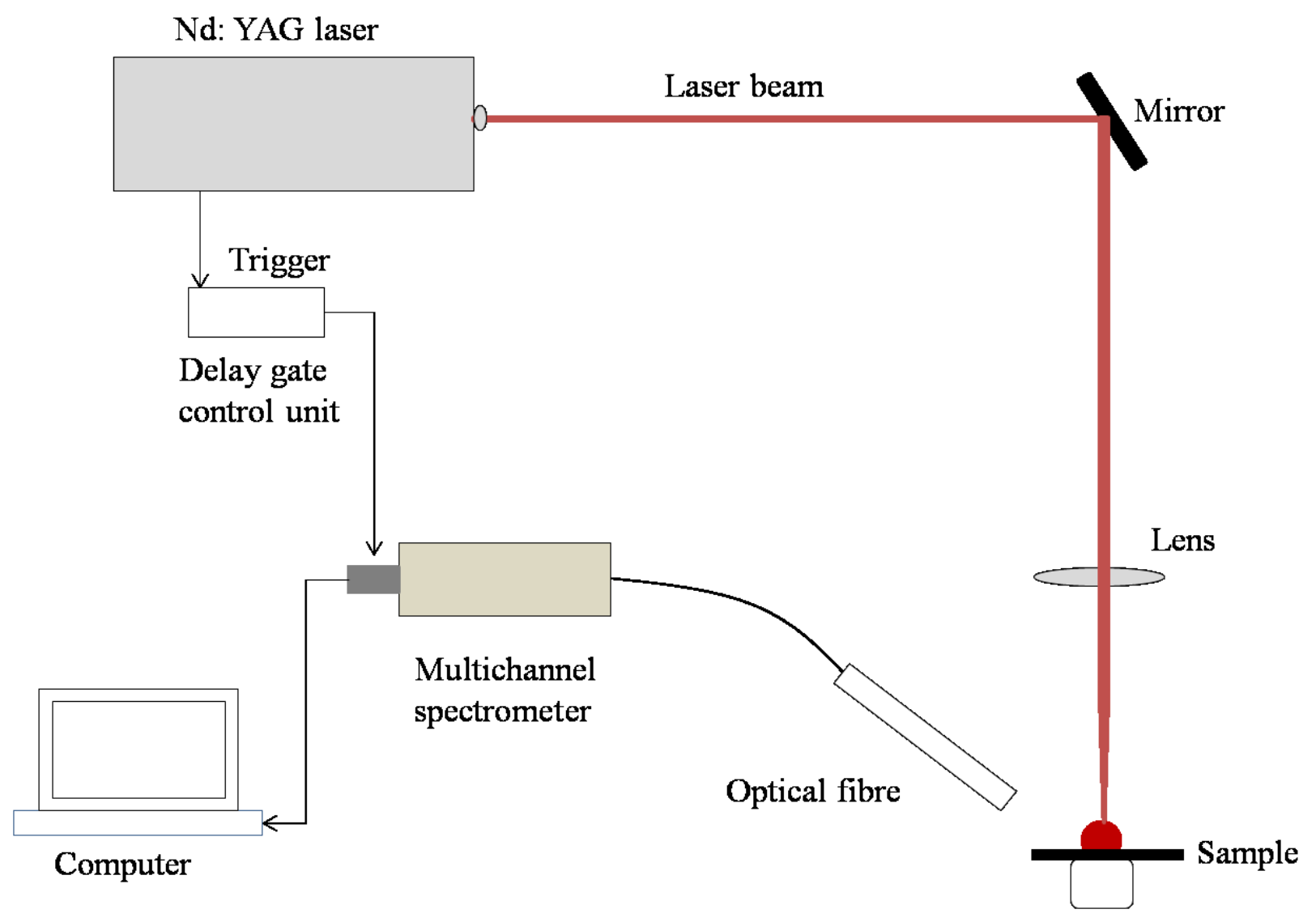

Fig. 1. Schematic diagram of the experimental set up.

\section{SAMPLE PROCESSING}

It should be noted here that the LIBS2500plus spectrometer don't have the capability of analyzing liquid sample. This means that we need to convert liquid water samples (under study) and standards samples into solid phase for analyzing them with LIBS. This was done by using the same procedure as described in [9]. The liquid water samples were taken from five different tanneries located in Gujrat and Sialkot and Narowal. All these samples were acetified with $\mathrm{HNO}_{3}$ till $\mathrm{pH}$ level dropped below two. We poured $0.5 \mathrm{ml}$ of sample on the absorption paper for solidification. ALBET (Germany) absorption papers were used for this purpose. Solidification of liquid standards samples was done by introducing small amount of liquid samples on absorption paper, followed by drying mechanism. More detail can be found in the ref [9]. The dried samples were then investigated with LIBS under optimized and well controlled experimental conditions.

\section{RESULTS AND DISCUSSION}

As discussed in section 2, that the LIBS system needs to be optimized for the generation more reliable and authentic results. First important parameter to be optimized is the laser 
pulse energy, since this is the main component in the experiment and is responsible for the emission of $\mathrm{X}$-rays.

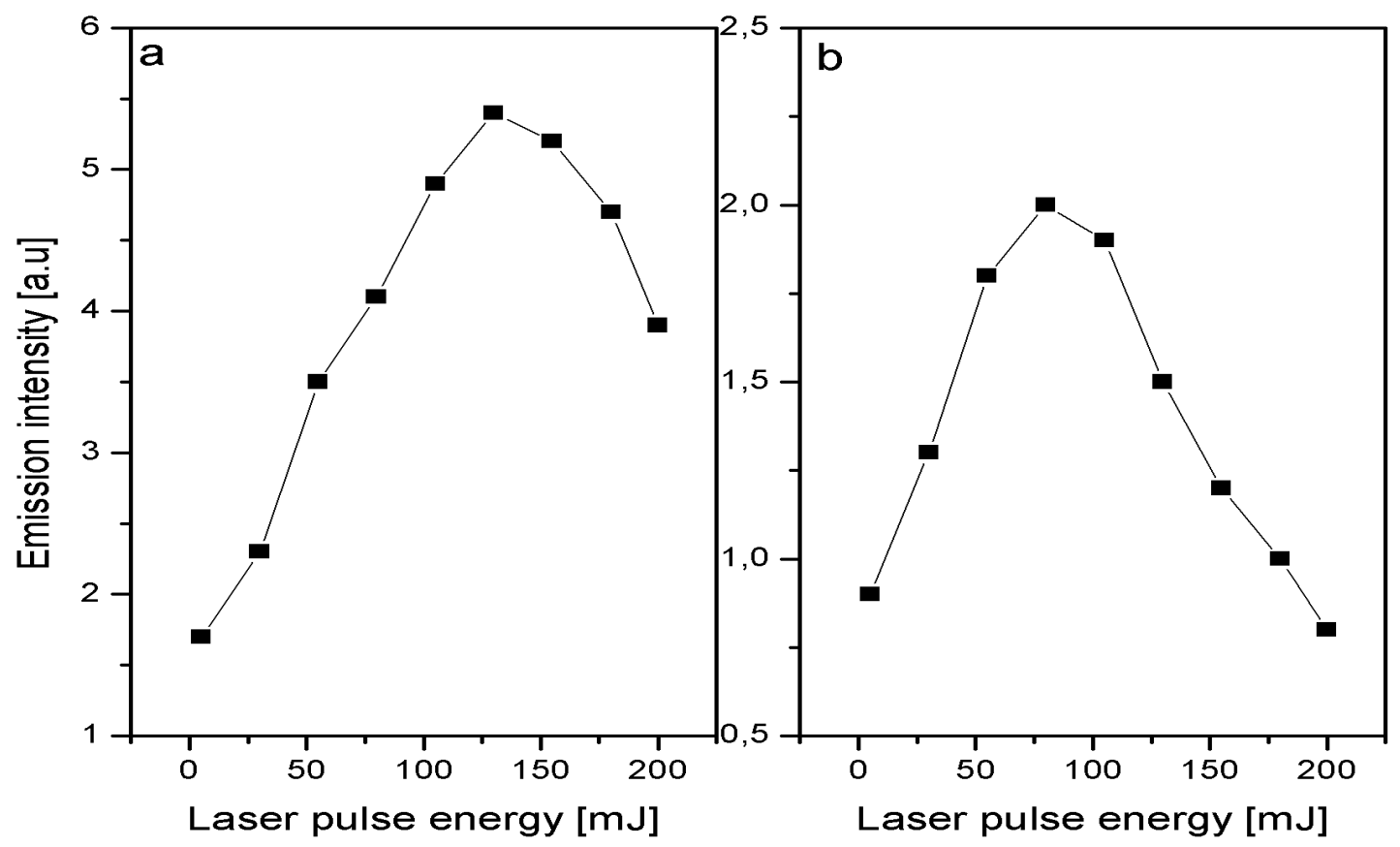

Fig. 2. a) Emission intensity of chromium (427.481 $\mathrm{nm}$ ) versus laser energy in $\mathrm{mJ}$, b) same as in (a) but for cadmium $(226.502 \mathrm{~nm})$.
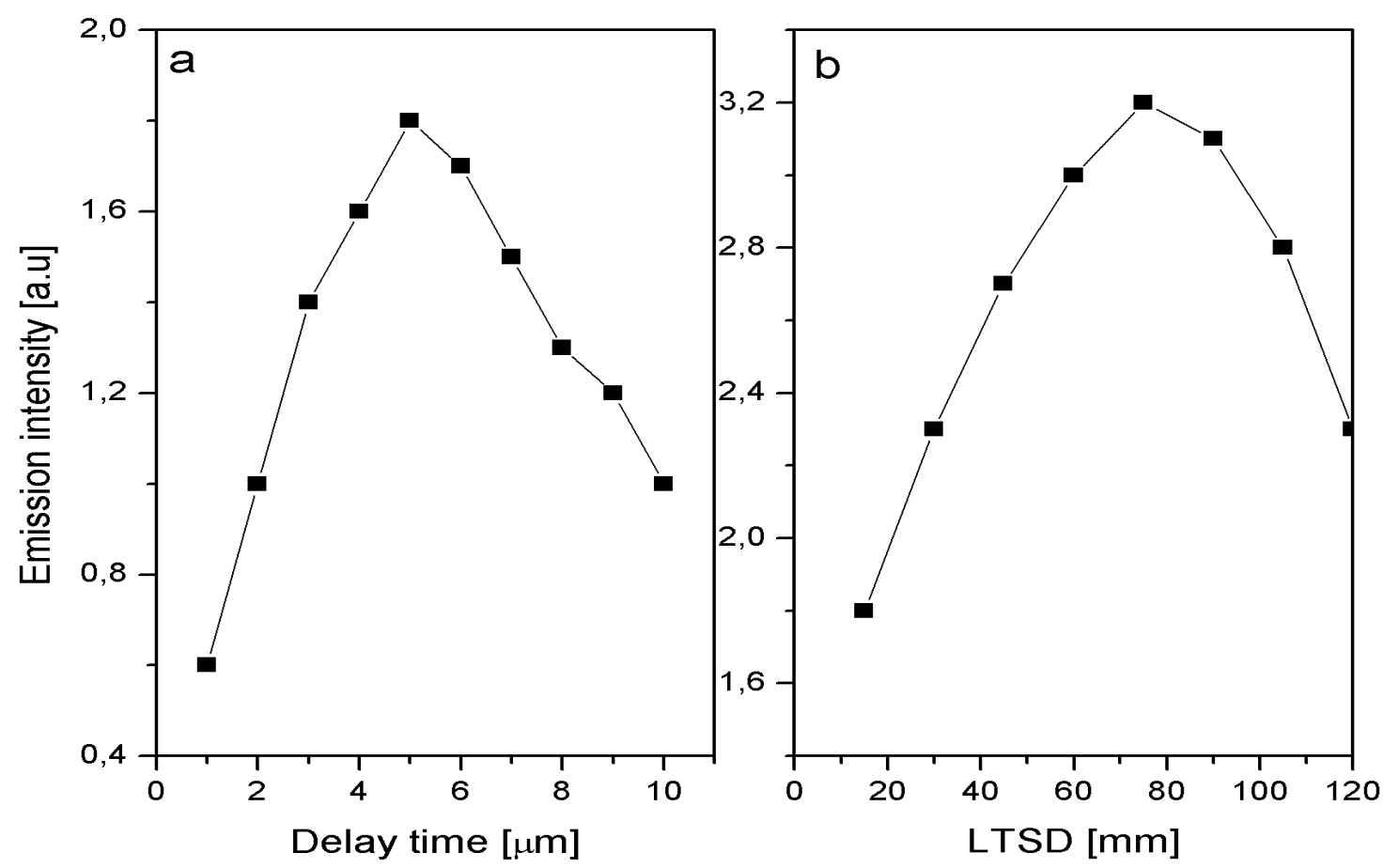

Fig. 3. a) Emission intensity versus delay time for chromium standard at $427.481 \mathrm{~nm}$, b) Emission intensity vs lens-to-sample distance (LTSD). 
In the present study we examine signal intensities as a function of laser pulse energy in the range of 5 to $200 \mathrm{~mJ}$ by keeping constant lens to surface distance and delay time. Fig. 2 shows laser pulse energy vs signal intensity for chromium standard at $226.502 \mathrm{~nm}$. It can be seen that the intensity of the emitted radiations increases with the increase of laser energy until the plasma density become to high where self-absorption and losses dominate the emission mechanism [10]. The optimised value of pulse energies obtained for chromium and cadmium are $75.2 \mathrm{~mJ}$ and $142.3 \mathrm{~mJ}$, respectively.

The result from the LIBS measurements are also very sensitive to the focusing lens-tosample distance (LTSD) and affect the mass of ablated material and emission line intensities. To avoid the occurrence of plasma shielding effects the laser beam energy is controlled in general and the LTSD is maintained few millimetres shorter than the lens focal length. In this work we have used lens with focal length of $100 \mathrm{~mm}$. We have investigated LTSD from 15 to $120 \mathrm{~mm}$ with different laser fluencies in order to avoid plasma shielding effects. The optimized value for LTSD found in the current study to be $\sim 70 \mathrm{~mm}$.
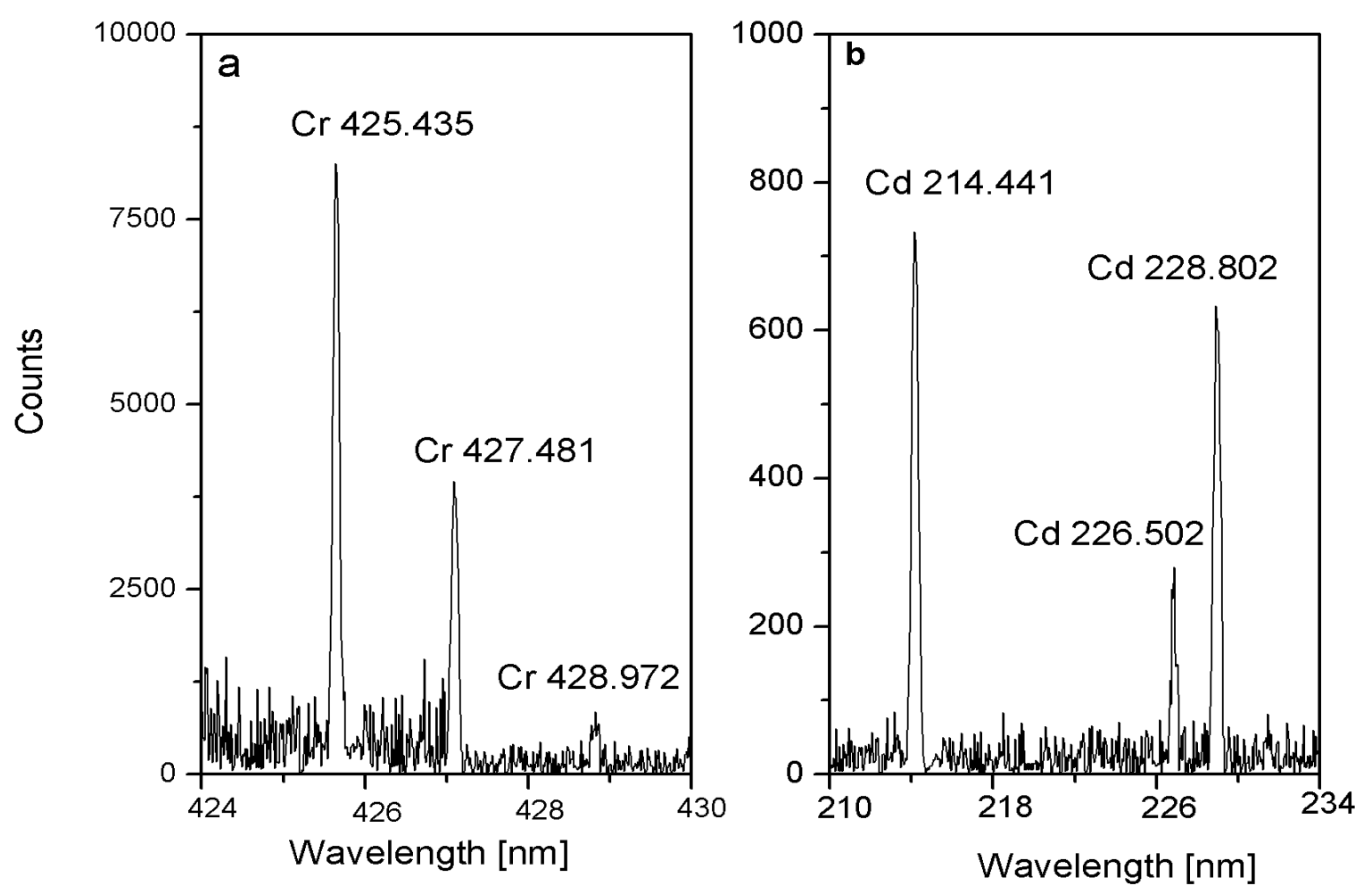

Fig. 4. High resolution LIBS spectra showing chromium and cadmium lines in Sialkot tannery wastewater sample.

The light collection system orientation and distance from the sample surface also play a crucial role in the measurements. The optimized angle and distance for fibre optics was found to be $\sim 45^{\circ}$ and $10 \mathrm{~mm}$, respectively. The fourth parameter is the delay time i.e. the time between firing the laser pulse and the opening of the camera shutter to collect light from the sample surface. Figure 4 shows that the maximum intensity is found to be at $3 \mu$ s for chromium standard peak at $226.502 \mathrm{~nm}$. 
From the high resolution spectra qualitative analysis were carried out with the aid of NIST data base [11] and found chromium and cadmium in all the samples. Figure 4 shows the LIBS spectra of chromium and cadmium from the tannery wastewater in Gujrat. For each metal present in the sample, average spectra of 30 laser shots were recorded under optimized condition of the LIBS parameters. The quantity of metal in each sample is determined in units of $\mathrm{mg} / \mathrm{L}$. The calibration equation as described in [9] was used to estimate the level of chromium and in the wastewater samples.

Table 1 shows the concentration of chromium and cadmium in the five samples. The abbreviations G, S and N denote that the samples are taken from Gujrat, Sialkot and Narowal, respectively.

Table. 1. Chromium and cadmium concentrations in different sample determined by LIBS and FAAS techniques. The concentration is given in ppm with error is given in square brackets

(standard deviation).

\begin{tabular}{|c|c|c|c|c|}
\hline & \multicolumn{2}{|c|}{ Chromium } & \multicolumn{2}{c|}{ Cadmium } \\
\hline Sample & LIBS & FAAS & LIBS & FAAS \\
\hline S-1-G & $312(07)$ & $327(0.2)$ & $472(11)$ & $455(0.6)$ \\
\hline S-2-G & $321(08)$ & $342(0.3)$ & $412(09)$ & $425(0.3)$ \\
\hline S-3-S & $473(09)$ & $455(0.5)$ & $513(13)$ & $569(0.7)$ \\
\hline S-4-S & $494(11)$ & $471(0.5)$ & $549(15)$ & $589(0.5)$ \\
\hline S-5-N & $320(06)$ & $311(0.2)$ & $352(07)$ & $312(0.2)$ \\
\hline
\end{tabular}

It can be seen that the results of chromium with LIBS and FAAS techniques for the samples S-1-G and S-2-G is around $300 \mathrm{mg} / \mathrm{L}$, for S-3-S and S-4-S is around $450 \mathrm{mg} / \mathrm{L}$ and for S-5-N is $320 \mathrm{mg} / \mathrm{L}$. The probable reason for the low concentration of chromium in Gujrat and Narowal samples compared to Sialkot samples is the less number of leather factories in these two cities. Since Sialkot is the main hub of producing leather garments and therefore have lot of factories which throw more waste in tanneries. The same behaviour can also be seen in case of cadmium. The level of cadmium is bit high compared to chromium due to the effluents carried cadmium-containing dyes and pigments.

\section{CONCLUSIONS}

With the present investigation it is concluded that the canneries water flowing through the factory areas is water is highly polluted with chromium and cadmium. The concentration of these metals varies in wastewater from sample to sample in three different cities depending on the strength of factories around the cannery from where sample is taken. Our LIBS and FAAS results agree well in all the samples. 


\section{References}

[1] V. S. Burakov et al., Spectrochimica Acta Part B 64 (2009) 141-146.

[2] K. N. Mekonnen, Bull. Chem. Soc. Ethiop. 27(1) (2013) 1-13.

[3] K. Song, Y. I. Lee, J. Sneddon, Applied Spectroscopy Reviews 37(1) (2002) 89.

[4] M. Yao et al., Applied Optics 51(10) (2012) 1552-1557.

[5] S. S. Tahir, R. Naseem, Separation and Purification Technology 53(3) (2007) 312-321.

[6] U. Rafique, A. Ashraf, A. K. Khan, S. Nasreen, R. Rashid, Q. Mahmood, Journal of the Chemical Society of Pakistan 32(5) (2010) 644-649.

[7] R. Gaudiuso et al., Sensors 10 (2010) 7434-7468; http://www.oceanoptics.com/Products/libs.asp

[8] M. Bukjari et al., Journal of Analytical Methods in Chemistry 10(1155) (2012) 823016.

[9] D. Giulietti, L. A. Gizzi, La Rivista del Nuovo Cimento 21 (1998) 1.

[10] Kramida, A., Ralchenko, Yu., Reader, J. and NIST ASD Team (2013). NIST Atomic Spectra Database (version 5.1), [Online]. Available: http://physics.nist.gov/asd 